\title{
Elemente und Ephemeride des Kometen 1910 b (Metcalf).
}

Die Elemente in A. N. 4440 wurden verbessert durch Anschiuß an eine Beobachtung vom 18. September in Bergedorf von Herrn Dr. Graff. Die neuen Elemente lauten:

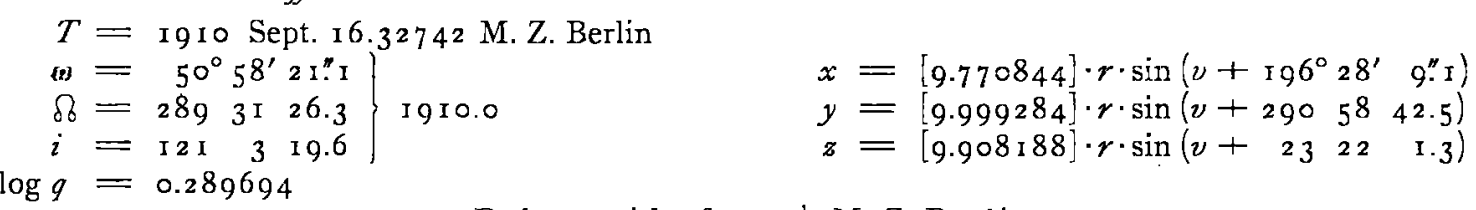

Ephemeride für $\mathbf{I}^{\text {h }}$ M. Z. Berlin.

\begin{tabular}{|c|c|c|c|c|c|}
\hline I9 ro & $\alpha$ I 910.0 & $\delta$ I 9 I 0.0 & $\log r$ & $\log d$ & Gr. \\
\hline Okt. I 2 & $\mathrm{I} 5^{\mathrm{h}} 26^{\mathrm{m}} 30^{\mathrm{s}}$ & $+18^{\circ} 37^{\prime} 6$ & 0.2955 & $0.4 \mathrm{I} 3 \mathrm{I}$ & I I ${ }^{\mathrm{m}} \cdot 8$ \\
\hline I 3 & $26 \quad 39$ & I $84 \mathrm{I} .5$ & & & \\
\hline I 4 & $26 \quad 48$ & 1845.6 & & & \\
\hline I 5 & $26 \quad 5^{8}$ & I 849.8 & & & \\
\hline 16 & 279 & I 854.1 & 0.2974 & 0.4188 & I I .8 \\
\hline I 7 & 2720 & $18 \quad 58.5$ & & & \\
\hline 18 & 2732 & 193.1 & & & \\
\hline 19 & 2745 & I 97.8 & & & \\
\hline 20 & $275^{8}$ & I 9 I 2.5 & 0.2996 & 0.4236 & I I .8 \\
\hline 2 I & $28 \quad 12$ & I $9 \quad 17.4$ & & & \\
\hline 22 & 2826 & I9 22.4 & & & \\
\hline 23 & $284 \mathrm{I}$ & 1927.6 & & & \\
\hline 24 & $285^{6}$ & 1932.9 & 0.3019 & 0.4276 & I I.9 \\
\hline 25 & 2912 & I $9 \quad 3^{8.3}$ & & & \\
\hline 26 & $29 \quad 29$ & rg 43.9 & & & \\
\hline 27 & 2946 & 1949.7 & & & \\
\hline 28 & 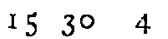 & +1955.6 & 0.3045 & 0.4308 & I 1.9 \\
\hline
\end{tabular}

\begin{tabular}{|c|c|c|c|c|c|c|}
\hline \multicolumn{2}{|c|}{ I9IO } & $\alpha$ I910.0 & $\delta$ rgro.0 & $\log r$ & $\log 1$ & Gr. \\
\hline Okt. & 28 & $15^{\mathrm{h}} 30^{\mathrm{m}} 4^{\mathrm{s}}$ & $+19^{\circ} 55^{\prime} 6$ & 0.3045 & 0.4308 & $\mathrm{I}^{\mathrm{m}} \cdot 9$ \\
\hline & 29 & $30 \quad 22$ & $20 \quad 1.6$ & & & \\
\hline & 30 & 3040 & 7.8 & & & \\
\hline & $3 \mathrm{I}$ & $30 \quad 59$ & 20 I 4.2 & & & \\
\hline Nov. & I & 3 I 18 & $20 \quad 20.7$ & 0.3073 & $0.433^{2}$ & I 1.9 \\
\hline & 2 & 3 I 38 & $20 \quad 27.4$ & & & \\
\hline & 3 & 3 I 58 & $20 \quad 34.3$ & & & \\
\hline & 4 & 3219 & $20 \quad 41 \cdot 3$ & & & \\
\hline & 5 & 3240 & $20 \quad 48.5$ & 0.3104 & $0.435^{\circ}$ & I 2.0 \\
\hline & 6 & $33 \quad I$ & $20 \quad 55.9$ & & & \\
\hline & 7 & 3322 & $2 \mathrm{I} \quad 3.6$ & & & \\
\hline & 8 & 3343 & 2 I I I.4 & & & \\
\hline & 9 & $34 \quad 4$ & $2 \mathrm{I} \quad \times 9.4$ & $0.3^{1} 3^{6}$ & 0.4359 & $\Upsilon 2.0$ \\
\hline & IO & 3426 & $21 \quad 27.6$ & & & \\
\hline & I I & $34 \quad 48$ & $2 \mathrm{I} \quad 3^{6 . \mathrm{I}}$ & & & \\
\hline & I 2 & 3510 & 2 I 44.7 & & & \\
\hline & I 3 & $15 \quad 3533$ & +2 I 53.5 & $0.3^{1} 70$ & $0.43^{6} \mathrm{I}$ & I 2.0 \\
\hline
\end{tabular}

Kiel, Bureau der Astr. Nachr., I 910 Okt. 9.

H. Kobold.

\section{Une variable nouvelle 95.1910 Leonis minoris.}

Ce 26 septembre Mme. L. Ceraski a trouvé une variable nouvelle dont voici les coordonnées approchées:

$$
\begin{aligned}
& \alpha=9^{\mathrm{h}} 45^{\mathrm{m}} 55^{\mathrm{s}} \quad d=+36^{\circ} 48^{\prime} \quad \text { (I855.0) } \\
& 9483^{8}+3^{6} 35 \text { (1900.0). }
\end{aligned}
$$

Moscou, r 9 ro sept. 30 .
A en juger par ${ }_{5} 5$ clichés, obtenus entre 1907 et I9ıo, l'éclat de cette étoile varie de $10^{\mathrm{m}}$ à $<\mathbf{I}^{\mathrm{m}}$.

M. S. Blažko ne trouve pas possible de se prononcer avec certitude sur le type de la variable.

Prof. W. Ceraski.

\section{Proiezione brillante su Saturno.}

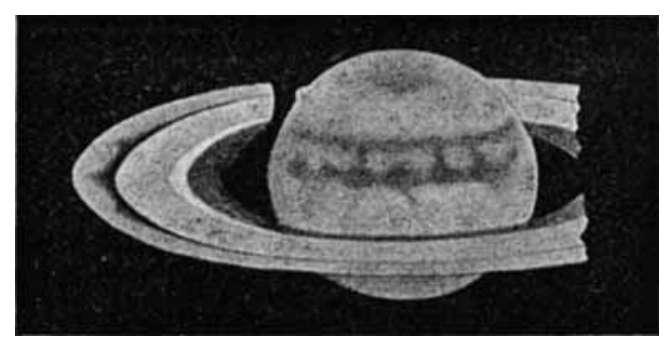

Il . 29 Settembre a $23^{\mathrm{h}} 3^{6^{\mathrm{m}}}$ (T. m. c. E. C.) ho osservato all'orlo W del globo di Saturno una prominenza luminosa, che si profilava sull'ombra portata del globo sull'anello.

La proiezione era in vicinanza di una larga macchia chiara, sfumata, al limite della banda equatoriale Sud.

Il fenomeno è stato osservato fino a $0^{\mathrm{h}} 20^{\mathrm{m}}$ del 30 Settembre, dopo non rimase visibile che la macchia chiara lungo il terminatore.

(Telescopio Calver di $35^{\circ} \mathrm{mm}$, Ingr. $35^{\circ}$ ).

Firenze, Osservatorio Ximeniano, r 9 ro Sett. 30.

Mentore Maggini.

In halt zu Nr. 4445. W. E. Raymond. Measures of Double Stars. 65. - H. Rosenberg. Über den Zusammenhang von Helligkeit und Spektraltypus in den Plejaden. 71. - Mitteilungen über kleine Planeten. 77. - L. Fabry. Ephéméride de la planète (444) Gyptis. 77. - H. Kobold. Elemente und Ephemeride des Kometen 1910 b (Metcalf). 79. - W. Ceraski. Une variable nouvelle 95.1910 Leonis minoris. 79. - M. Maggini. Proiezione brillante su Saturno. 79. 